\title{
氟添加方式对NiMo催化剂在浆态床上加氢脱硫性能的影响
}

\author{
石 林 黄 伟* 宋书征 李志博 刘双强 \\ (太原理工大学, 煤科学与技术教育部和山西省重点实验室, 太原 030024)
}

\begin{abstract}
摘要: 以 $\mathrm{F}$ 为助剂, 采用完全液相法制备了氟改性的 $\mathrm{NiMo} / \mathrm{TiO}_{2}-\mathrm{Al}_{2} \mathrm{O}_{3}$ 浆状催化剂, 考察了氟的添加方式对催化 剂在浆态床上4,6-二甲基二苯并噻吩(4,6-DMDBT)加氢脱硫性能的影响. 采用X射线衍射 (XRD)谱、氢气程序 升温还原 $\left(\mathrm{H}_{2}-\mathrm{TPR}\right) 、 \mathrm{~N}_{2}$ 吸脱附实验 $(B E T) 、 X$ 射线光电子能谱 $(X P S)$ 、高分辨率透射电镜( $\left.H R T E M\right)$ 对催化剂进 行了表征. 结果表明, 在不添加浓硝酸的情况下, 在活性组分加入之前引入氟能显著提高催化剂的比表面积和 孔径, 促进反应过程中金属镍在催化剂表面的分散, 更大程度地减弱催化剂中活性金属与载体间的相互作用, 有效提高Mo的硫化度及 $\mathrm{MoS}_{2}$ 的堆积层数, 从而生成了较多的II类Ni-Mo-S活性相, 促进芳香环的加氢和 $\mathrm{C}-\mathrm{S}$ 键的氢解, 对 4,6-DMDBT有较高的加氢脱硫活性.
\end{abstract}

关键词：完全液相法；氟； 4,6-二甲基二苯并噻吩； 浆态床

中图分类号: 0643

\section{Effect of the Fluorine Addition Method on the Hydrodesulfurization Performance of NiMo Catalysts over Slurry Bed}

\author{
SHI Lin HUANG Wei ${ }^{*} \quad$ SONG Shu-Zheng LI Zhi-Bo LIU Shuang-Qiang \\ (Key Laboratory of Coal Science and Technology of Ministry of Education and Shanxi Province, Taiyuan University of \\ Technology, Taiyuan 030024, P. R. China)
}

\begin{abstract}
NiMo} / \mathrm{TiO}_{2}-\mathrm{Al}_{2} \mathrm{O}_{3}$ slurry catalysts with fluorine as an additive were prepared by complete liquidphase technology for hydrodesulfurization. The effect of different fluorine addition methods on the properties of the catalysts for 4,6-dimethyldibenzothiophene (4,6-DMDBT) hydrodesulfurization were investigated. The catalysts were characterized by X-ray diffraction (XRD), temperature-programmed reduction of $\mathrm{H}_{2}\left(\mathrm{H}_{2}-\mathrm{TPR}\right), \mathrm{N}_{2}$ adsorption-desorption isotherms (BET), X-ray photoelectron spectroscopy (XPS), and high-resolution transmission electron microscopy (HRTEM). The results reveal that in the absence of nitric acid, adding fluoride into the catalyst before the introduce of molybdenum and nickel ions can significantly increase the surface area and average pore size, improve the dispersion of metallic nickel on the surface of catalyst, and weaken the interaction between the metal and the support. This effectively increases the sulfidation degree of $\mathrm{Mo}, \mathrm{MoS}_{2}$ slab stacking, and the content of the highly active Ni-Mo-S(II) phase, which can promote the hydrogenation of the aromatic ring and the hydrogenolysis of the $\mathrm{C}-\mathrm{S}$ bond, and thus increase the hydrodesulfurization activity for 4,6-DMDBT.
\end{abstract}

Key Words: Complete liquid-phase technology; Fluoride; 4, 6-DMDBT; Slurry bed

Received: March 18, 2015; Revised: June 5, 2015; Published on Web: June 8, 2015.

"Corresponding author. Email: huangwei@tyut.edu.com; Tel: +86-351-6018073.

The project was supported by the National Natural Science Foundation of China (21336006), General Program of National Natural Science Foundation of China (21176176), and Research Fund for the Doctoral Program of Higher Education of China (20111402130002).

国家自然科学基金重点项目(21336006), 国家自然科学基金面上项目(21176176)和高等学校博士学科点专项(优先发展领域)(20111402130002) 资助

(C) Editorial office of Acta Physico-Chimica Sinica 


\section{1 引言}

随着世界范围内车辆柴油化的不断加快, 柴油 的需求量越来越大. 柴油中含有硫和氮的化合物, 燃烧后排放出的 $\mathrm{SO}_{r}$ 和 $\mathrm{NO}_{r}$ 是形成PM2.5的重要原因 之一. 因此, 加快推进油品质量升级, 降低柴油中 硫、氮含量就成为当务之急 ${ }^{1,2}$ 为了保护日益严峻 的大气环境, 国内外都制定了更为苛刻的柴油标准. 我国制定的GB19147-2013《车用柴油(IV)》标准 也在2013年2月7日发布并实施，过渡期至2014年 12月 31 日, 从2015年1月1日起全面实施(该标准要求 柴油生产、储运及使用各环节都要满足硫含量低 于 $50 \mu \mathrm{g} \cdot \mathrm{g}^{-1}$ 的要求). ${ }^{3}$ 但是, 目前我国许多市售柴油 的硫含量不达标, 严重影响了国 IV 标准的执行.

加氢脱硫是生产清洁柴油的重要技术, 目前相 关的研究主要集中在固定床反应器. 4,5 固定床反应 器由于传热不高, 易引起局部过热, 进而导致催化 剂的积炭和失活. 浆态床具有传热效率高、易于恒 温操作、能量利用率高等优点, 近几年成为加氢领 域的重要发展方向, 特别适用于强放热反应. 加氢 脱硫为强放热反应，催化剂用量大，浆态床高效的 传热优点在该体系中将体现得更为突出. ${ }^{6,7}$ 本课题 组针对浆态床催化剂使用特性, 提出了从溶液到浆 液直接制备浆态床催化剂的完全液相方法, ${ }^{8,9}$ 该方 法制备的加氢脱硫催化剂对 4,6-DMDBT显示出很 高的脱硫活性, 但催化剂重现性差. ${ }^{10}$ 本文在本课题 组前期研究基础上, 针对完全液相NiMo催化剂由于 反应原料受周围环境影响大, 催化剂表面性质及活 性组分在催化剂表面分散不稳定和重现性差等问 题, 提出了用氟改性NiMo催化剂.

许多研究表明, 在催化剂中添加第二助剂往往 是改善催化剂活性及稳定性最经济有效的方法. 氟 是常用的添加剂之一, ${ }^{11-13}$ 氟改性催化剂的加氢脱硫 活性与氟的添加方式有很大关系. Matralis等 ${ }^{14}$ 认为, 在F-Co-Mo $/ \gamma-\mathrm{Al}_{2} \mathrm{O}_{3}$ 催化剂中氟在活性组分后浸渍 虽减小了催化剂的比表面积, 但促进了活性组分的 分散, 增加了反应活性位数量, 进而增加了加氢脱 硫的活性; Lewis等 ${ }^{15}$ 认为, 在 $\mathrm{Ni}-\mathrm{Mo} / \mathrm{Al}_{2} \mathrm{O}_{3}$ 催化剂中 氟与活性组分同时浸渍可以得到活性较高的催化 剂; Sarbak和Andersson ${ }^{16}$ 发现在 $\mathrm{CoMo} / \mathrm{Al}_{2} \mathrm{O}_{3}$ 催化剂 中氟与活性组分的浸渍顺序对催化剂影响较小. 因 此, 本文重点研究了在完全液相法工艺中氟添加方 式对催化剂性能的影响, 以期了解完全液相工艺中 氟助剂的作用规律.

\section{2 实验部分}

\section{1 催化剂的制备}

完全液相法制备的催化剂都是先通过溶胶-凝 胶法制得催化剂前驱体, 然后在室温老化9-10 d形 成凝胶, 最后将凝胶分散于液体石蜡中 $280{ }^{\circ} \mathrm{C}$ 热处 理 $8 \mathrm{~h}$ 得浆状催化剂. 催化剂前驱体制备: 将一定量 异丙醇铝溶于适量异丙醇中, 在 $82.5{ }^{\circ} \mathrm{C}$ 的恒温水浴 锅中回流摚拌 $3 \mathrm{~h}$, 醇解液常温下放置 $12 \mathrm{~h}$; 强烈摚 拌下, 将异丙醇钛、异丙醇和冰乙酸的混合液逐滴 滴加到醇解液中, 滴加完毕后将水浴锅温度调到 85 ${ }^{\circ} \mathrm{C}$. 待其温度升到 $85{ }^{\circ} \mathrm{C}$ 时, 将 $330 \mathrm{~mL}$ 蒸馏水用蠕动 泵逐滴滴加到溶液中 (滴加速率为 $5.5 \mathrm{~mL} \cdot \mathrm{min}^{-1}$ ), 滴 完后 $85{ }^{\circ} \mathrm{C}$ 恒温摚拌 $1 \mathrm{~h}$, 然后采用以下 4 种加料方式 来制备催化剂前驱体(加料时间间隔均为 $1 \mathrm{~h}$ ): (1)依 次滴加一定量的浓 $\mathrm{HNO}_{3}(65 \%-68 \%) 、 \mathrm{Ni}\left(\mathrm{NO}_{3}\right)_{2} \cdot 6 \mathrm{H}_{2} \mathrm{O}$ (AR, 国药集团化学试剂有限公司)的异丙醇溶液、 $\left(\mathrm{NH}_{4}\right)_{6} \mathrm{Mo}_{7} \mathrm{O}_{24} \cdot 4 \mathrm{H}_{2} \mathrm{O}(\mathrm{AR}$, 国药集团化学试剂有限公 司)水溶液, 所得催化剂记为CAT1; (2)依次滴加浓 $\mathrm{HNO}_{3} 、 \mathrm{Ni}\left(\mathrm{NO}_{3}\right)_{2} \cdot 6 \mathrm{H}_{2} \mathrm{O}$ 的异丙醇溶液、 $\left(\mathrm{NH}_{4}\right)_{6} \mathrm{Mo}_{7} \mathrm{O}_{24} \cdot$ $4 \mathrm{H}_{2} \mathrm{O}$ 水溶液、 $\mathrm{NH}_{4} \mathrm{~F}(\mathrm{AR}$, 天津市光复科技发展有限 公司)水溶液, 所得催化剂记为CAT2; (3)依次滴加浓 $\mathrm{HNO}_{3} 、 \mathrm{NH}_{4} \mathrm{~F}$ 水溶液、 $\mathrm{Ni}\left(\mathrm{NO}_{3}\right)_{2} \cdot 6 \mathrm{H}_{2} \mathrm{O}$ 的异丙醇溶 液、 $\left(\mathrm{NH}_{4}\right)_{6} \mathrm{Mo}_{7} \mathrm{O}_{24} \cdot 4 \mathrm{H}_{2} \mathrm{O}$ 水溶液, 所得催化剂记为 CAT3; (4)依次滴加 $\mathrm{NH}_{4} \mathrm{~F}$ 水溶液、 $\mathrm{Ni}\left(\mathrm{NO}_{3}\right)_{2} \cdot 6 \mathrm{H}_{2} \mathrm{O}$ 的 异丙醇溶液、 $\left(\mathrm{NH}_{4}\right)_{6} \mathrm{Mo}_{7} \mathrm{O}_{24} \cdot 4 \mathrm{H}_{2} \mathrm{O}$ 水溶液, 所得催化 剂记为CAT4. 以上 4 个催化剂中, 摩尔比 $n(\mathrm{Ni}) / n(\mathrm{Ni}+$ $\mathrm{Mo})=0.43, n(\mathrm{Ti}) / n(\mathrm{Al})=0.25$. 氟改性催化剂中氟的 添加量为 $3 \%(w)$.

\section{2 催化剂表征}

完全液相法制备的催化剂初始形态均为浆状, 在表征测试前取静置后的烧杯底部浆状催化剂若 干克, 放在索式提取器用石油醚抽提 $2 \mathrm{~d}$, 然后室温 下放置使溶剂挥发, 自然干燥后进行各项性能表征.

样品的XRD表征采用日本理学 $\mathrm{D} / \max 2500$ 型 粉末 $\mathrm{X}$ 射线衍射仪, $\mathrm{Cu} K_{a}$ 辐射源, $\mathrm{Ni}$ 滤片, 管电压为 $40 \mathrm{kV}$, 管电流为 $40 \mathrm{~mA}$, 扫描范围 $10^{\circ}-85^{\circ}$, 扫描速 率4 $\left.{ }^{\circ}\right) \cdot \min ^{-1}$.

样品的BET表征采用美国Quantachrome SI系列 吸附仪于 $-196^{\circ} \mathrm{C}$ 进行 $\mathrm{N}_{2}$ 吸附测定. 根据BET计算催 化剂的比表面积, 采用BJH模型处理低温吸附等温 线得到孔径和孔容.

样品的TPR表征采用TP-5000型多用吸附仪(天 津先权仪器厂), 催化剂用量为 $100 \mathrm{mg}$, 以体积分数 
为 $5 \% \mathrm{H}_{2}-95 \% \mathrm{~N}_{2}$ 的混合气为还原气，流速为 30 $\mathrm{mL} \cdot \mathrm{min}^{-1}$, 以 $10^{\circ} \mathrm{C} \cdot \mathrm{min}^{-1}$ 速率升温, 热导检测耗氢量, 记录TPR曲线.

样品XPS表征采用AXISULTRA DLD 光电子 能谱仪(Kratos公司生产)进行XPS检测, 半球状电子 分析仪, 激光源为单色器 $\mathrm{Al}$ 靶 $(h v=1486.6 \mathrm{eV})$, 以 $\mathrm{C} s 1\left(E_{\mathrm{B}}=284.6 \mathrm{eV}\right)$ 为标准校正核电效应.

样品的HRTEM分析采用FEITECNAL G2F20型 透射电镜, 点分辨率 $0.248 \mathrm{~nm}$, 线分辨率 $0.102 \mathrm{~nm}$, 放大倍数达 105 万倍.

\section{3 催化剂活性评价}

称取适量的4,6-DMDBT加入到 $80 \mathrm{~mL}$ 液体石蜡 中加热搅拌使其完全溶解, 得到硫含量为 374.4 $\mu \mathrm{g} \cdot \mathrm{g}^{-1}$ 的样品, 将该样品与制备的 $20 \mathrm{~mL}$ 浆状催化剂 混合均匀后装入 $250 \mathrm{~mL}$ 反应釜中; $\mathrm{N}_{2}$ 吹扫 $1 \mathrm{~min}$, 通 入 $\mathrm{H}_{2}$ 至压力为 $2.5 \mathrm{MPa}$; 待温度升至 $350{ }^{\circ} \mathrm{C}$ 时压力为 $4 \mathrm{MPa}$, 反应 $6 \mathrm{~h}$. 脱硫率计算如下:

$$
x=\frac{w(\mathrm{~S})_{\mathrm{a}}-w(\mathrm{~S})_{\mathrm{b}}}{w(\mathrm{~S})_{\mathrm{a}}} \times 100 \%
$$

式中 $x$ 为总硫转化率, $w(\mathrm{~S})_{\mathrm{a}} 、 w(\mathrm{~S})_{\mathrm{b}}$ 为反应前后样品 的硫含量, 单位为 $\mu \mathrm{g} \cdot \mathrm{g}^{-1}$.

\section{3 结果与讨论}

\section{1 催化剂表征}

\subsubsection{XRD表征}

图1为催化剂反应前后的XRD谱图. 由图1可以 看出, 反应前 4 个催化剂均只有 $\mathrm{AlO}(\mathrm{OH})$ 的衍射峰, 没有出现明显的 $\mathrm{TiO}_{2} 、 \mathrm{MoO}_{3} 、 \mathrm{NiO}$ 的特征衍射峰, 说明这3种物质的聚集态没有达到XRD的可检测量,
说明组分分散性较好. 反应后 4 个催化剂也均未发 现有 $\mathrm{MoS}_{2}$ 的特征衍射峰, 说明 $\mathrm{MoS}_{2}$ 优良的分散状 态, 这与完全液相制备工艺有直接关系. 反应后, 催 化剂在 $2 \theta$ 为 $44.3^{\circ}$ 和 $51.7^{\circ}$ 出现金属 $\mathrm{Ni}$ 的特征衍射 峰, ${ }^{17}$ 强度顺序为 CAT $1 \approx \mathrm{CAT} 2>\mathrm{CAT} 3>\mathrm{CAT} 4$; 根据Scherrer公式计算的金属Ni的晶粒尺寸大小顺 序为CAT2 $22.75 \mathrm{~nm})>\operatorname{CAT} 1(18.29 \mathrm{~nm})>$ CAT3 $(16.71 \mathrm{~nm})>\operatorname{CAT} 4(16.51 \mathrm{~nm})$. 说明CAT4中 金属 $\mathrm{Ni}$ 晶粒尺寸最小, 分散性最好.

\subsubsection{BET表征}

图2为反应前后催化剂的氮气吸脱附等温曲线 及孔径分布曲线, 表 1 为织构参数. 从图 2 可以看出, 4 个催化剂反应前后的氮吸脱附曲线均属于IV型, 18,19 具有良好的介孔特征. 催化剂中引入F后, 吸脱附曲 线上移, 表明比表面积增大; 滞后环在CAT4显著变 宽, 说明介孔发达. 孔分布曲线显示, F的加入使催 化剂的大孔增多, 其中CAT4孔径分布明显变宽, 大 孔明显增多, 说明 $\mathrm{F}$ 的加入方式对催化剂孔径分布 有明显影响.

\subsubsection{TPR表征}

图3 是催化剂反应前的TPR谱图. 根据文献, ${ }^{18,20}$ 低温处的还原峰为高度分散的八面体配位的多层 $\mathrm{Mo}$ 物种的还原, 高温还原峰为四面体配位的单层 $\mathrm{Mo}$ 物种的还原及Mo氧化物的进一步还原. 由图3可 以看出, 加F后低温还原峰温略微向高温迁移, 高温 还原峰温向低温迁移, 其中CAT2和CAT3迁移较大. 这是由于 $F$ 的引入取代了载体表面上的部分羟基, $\mathrm{Mo}$ 物种与载体的作用力变小, 抑制了四面体配位 $\mathrm{Mo}$ 物种与 $\mathrm{Al}$ 结合生成较难还原的Mo-O-Al物种, 取

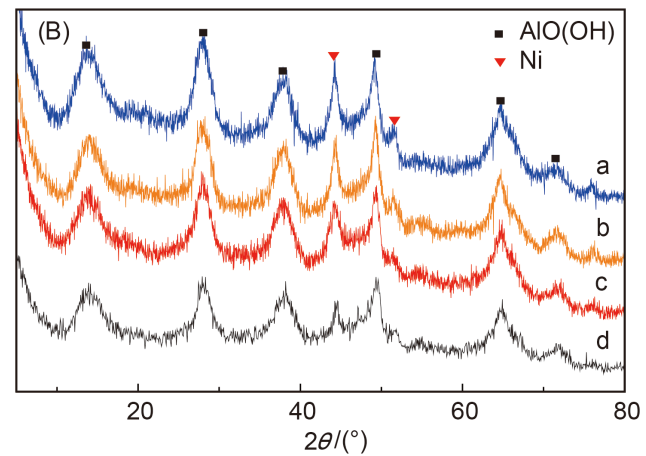

图1 催化剂反应前(A)后(B)的XRD谱图

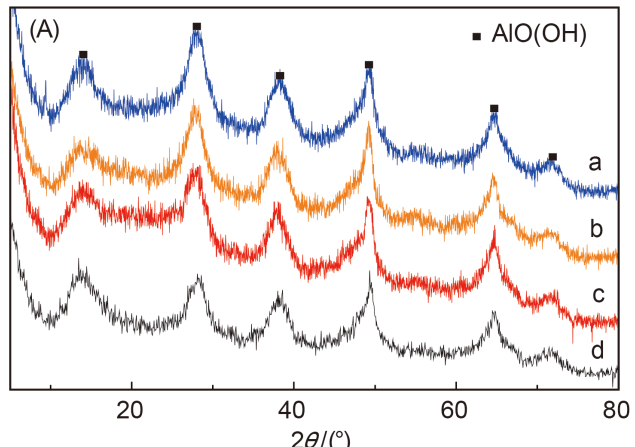

Fig.1 XRD patterns of catalysts before (A) and after (B) reaction

(a) CAT1; (b) CAT2; (c) CAT3; (d) CAT4. Four kinds of catalyst precursors were prepared by different methods of adding material. CAT1: adding $\mathrm{HNO}_{3}, \mathrm{Ni}\left(\mathrm{NO}_{3}\right)_{2} \cdot 6 \mathrm{H}_{2} \mathrm{O},\left(\mathrm{NH}_{4}\right)_{6} \mathrm{Mo}_{7} \mathrm{O}_{24} \cdot 4 \mathrm{H}_{2} \mathrm{O}$ in turn; $\mathrm{CAT} 2$ : adding $\mathrm{HNO}_{3}, \mathrm{Ni}\left(\mathrm{NO}_{3}\right)_{2} \cdot 6 \mathrm{H}_{2} \mathrm{O},\left(\mathrm{NH}_{4}\right)_{6} \mathrm{Mo}_{7} \mathrm{O}_{24} \cdot 4 \mathrm{H}_{2} \mathrm{O}, \mathrm{NH}_{4} \mathrm{~F}$ in turn; CAT3: adding $\mathrm{HNO}$, $\mathrm{NH}_{4} \mathrm{~F}, \mathrm{Ni}\left(\mathrm{NO}_{3}\right)_{2} \cdot 6 \mathrm{H}_{2} \mathrm{O},\left(\mathrm{NH}_{4}\right)_{6} \mathrm{Mo}_{7} \mathrm{O}_{24} \cdot 4 \mathrm{H}_{2} \mathrm{O}$ in turn; CAT4: adding $\mathrm{NH}_{4} \mathrm{~F}, \mathrm{Ni}\left(\mathrm{NO}_{3}\right)_{2} \cdot 6 \mathrm{H}_{2} \mathrm{O},\left(\mathrm{NH}_{4}\right)_{6} \mathrm{Mo}_{7} \mathrm{O}_{24} \cdot 4 \mathrm{H}_{2} \mathrm{O}$ in turn 

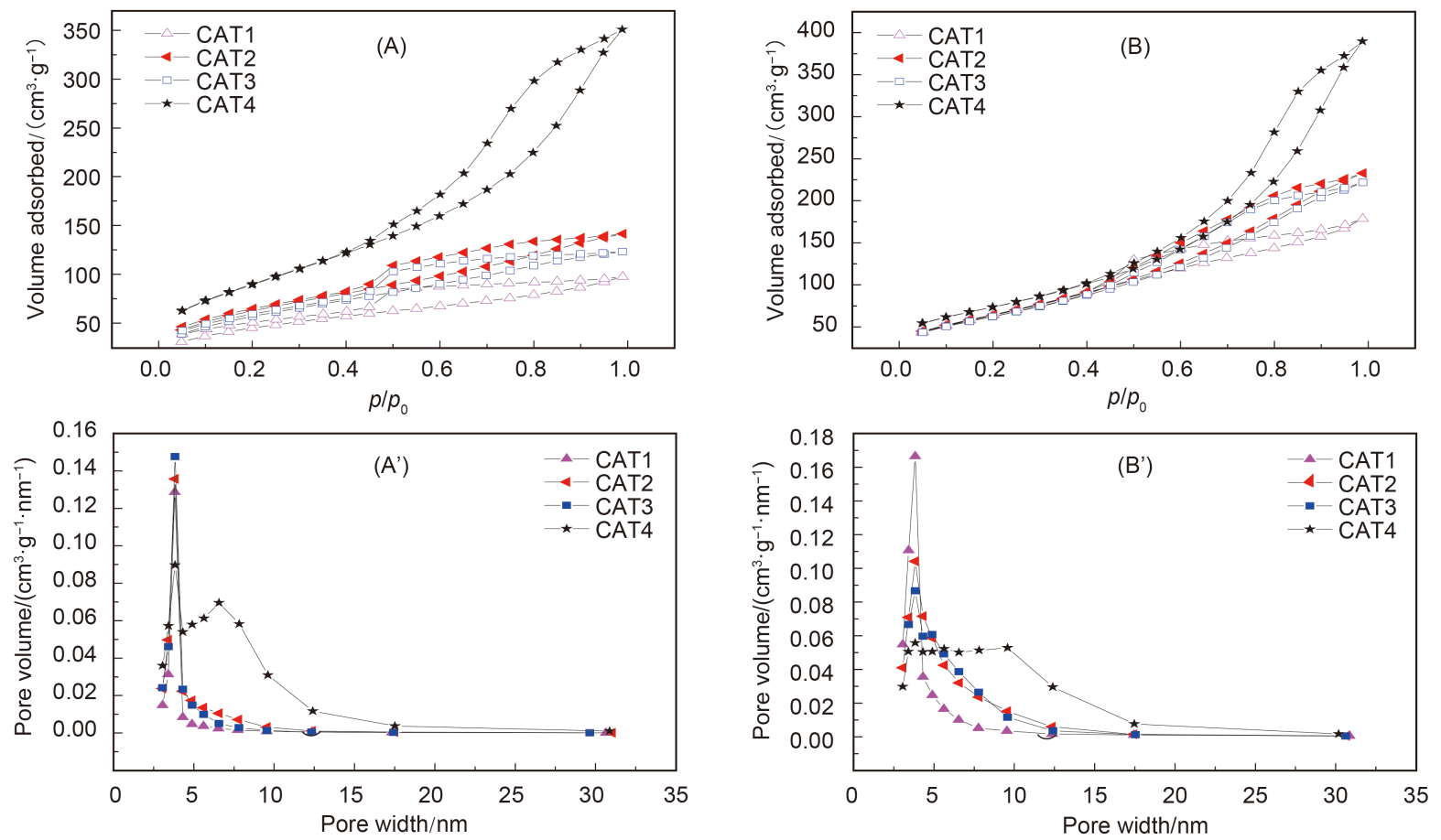

图2 催化剂反应前 $\left(A, A^{\prime}\right)$ 后 $\left(B, B^{\prime}\right)$ 的 $N_{2}$ 吸脱附曲线 $(A, B)$ 及孔径分布 $\left(A^{\prime}, B^{\prime}\right)$ 图

Fig.2 $\mathrm{N}_{2}$ adsorption-desorption isotherms $(\mathrm{A}, \mathrm{B})$ and $\mathrm{BJH}$ pore size distributions $\left(\mathrm{A}^{\prime}, \mathrm{B}^{\prime}\right)$ of catalysts before $\left(A, A^{\prime}\right)$ and after $\left(B, B^{\prime}\right)$ reaction

而代之的是生成了更多的Mo-O-Mo物种(即聚合态 的八面体Mo物种), 同时使部分高度分散的Mo物种

\section{表1 催化剂的织构参数}

Table 1 Textural and structural characteristics of catalysts

\begin{tabular}{|c|c|c|c|c|c|c|}
\hline \multirow{2}{*}{ Catalyst } & \multicolumn{3}{|c|}{ Before reaction } & \multicolumn{3}{|c|}{ After reaction } \\
\hline & $S_{\mathrm{BET}} /\left(\mathrm{m}^{2} \cdot \mathrm{g}^{-}\right.$ & $V_{\mathrm{p}} /(\mathrm{mL} \cdot \mathrm{g}$ & $D_{\mathrm{p}} / \mathrm{nm}$ & $S_{\mathrm{BET}} /\left(\mathrm{m}^{2} \cdot \mathrm{g}\right.$ & $V_{\mathrm{p}} /(\mathrm{mL} \cdot \mathrm{g}$ & $D_{\mathrm{p}} / \mathrm{nm}$ \\
\hline CAT1 & 164 & 0.151 & 3.69 & 243 & 0.276 & 4.54 \\
\hline CAT2 & 228 & 0.219 & 3.84 & 242 & 0.360 & 5.96 \\
\hline CAT3 & 209 & 0.191 & 3.63 & 235 & 0.343 & 5.85 \\
\hline CAT4 & 334 & 0.542 & 6.50 & 269 & 0.602 & 8.97 \\
\hline
\end{tabular}

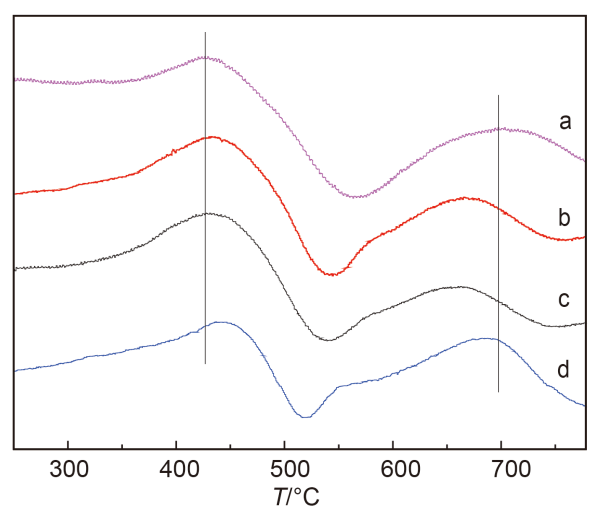

图3 催化剂反应前的 $\mathrm{H}_{2}-\mathrm{TPR}$ 曲线

Fig.3 $\quad \mathrm{H}_{2}$-TPR profiles of catalysts before reaction (a) CAT1; (b) CAT2; (c) CAT3; (d) CAT4
转化为聚合态的Mo物种, 导致Mo分散度略微降低, 颗粒略微变大. ${ }^{21}$ 特别值得注意的是, 加 $\mathrm{F}$ 后在 $550-$ $600{ }^{\circ} \mathrm{C}$ 之间出现了一个肩峰, 该峰是 $\mathrm{Ni}^{2+}$ 与部分聚合 态的Mo物种相互作用的结果. 显然, F的加入虽减弱 了 $\mathrm{Ni}$ 与载体的相互作用, 但却强化了 $\mathrm{Ni}^{2+}$ 与聚合态 的Mo物种的作用, 出现了分散状态的 $\mathrm{Ni}^{2+}$. 文献 ${ }^{20-22}$ 认为, 分散状态的 $\mathrm{Ni}^{2+}$ 与聚合态的 $\mathrm{Mo}$ 物种的存在有 利于催化剂在反应过程中生成高活性的II类Ni-MoS活性相. CAT4中肩峰最为明显, 还原温度最低, 映 证了CAT4中存在更多的 $\mathrm{Ni}^{2+}$ 与部分聚合态的 $\mathrm{Mo}$ 相 互作用的物种，更多的II类Ni-Mo-S活性相被生成， 因而脱硫效率更高.

\subsubsection{XPS表征}

表2 和表 3 为XPS测得的反应前后催化剂表面元 素组成. 反应前后, 加 $\mathrm{F}$ 催化剂表面 $\mathrm{C}$ 含量均比不加 $\mathrm{F}$ 催化剂要低, 表明 $\mathrm{F}$ 的引入对催化剂的表面性质有 很大改变, 表面亲水性增加, 这与 $\mathrm{F}$ 的高电负性一致. 反应后, 表面 $\mathrm{C}$ 含量进一步降低, 是反应条件下催化 剂表面态进一步微调的结果. 从各催化剂表面元素 组成看, 不加 $F$ 的催化剂 CAT1表面 $\mathrm{Ni} /(\mathrm{Ni}+\mathrm{Mo})$ 摩尔 比接近投料组成 $[n(\mathrm{Ni}) / n(\mathrm{Ni}+\mathrm{Mo})=0.43]$, 表明用 课题组完全液相法制备的催化剂组成较为均匀. 对 于加F催化剂, 无论反应前还是反应后, 其表面 $\mathrm{Ni}$ / 
$(\mathrm{Ni}+\mathrm{Mo})$ 摩尔比均大于不加 $\mathrm{F}$ 的催化剂, 说明 $\mathrm{F}$ 的引 入能促进 $\mathrm{Ni}$ 在催化剂表面的富集. 反应后 CAT1、 CAT2、CAT3的 $\mathrm{Ni} /(\mathrm{Ni}+\mathrm{Mo})$ 摩尔比均较反应前有所 降低, CAT4有所升高, 表明 $\mathrm{Ni}$ 在聚合态的Mo物种中 的分散度有所提高, ${ }^{18}$ 说明F的引入方式对催化剂在 反应过程中的表面态微调有重要影响. 这或许是 CAT4具有最高活性的主要原因之一. 比较表2和表 3 中反应前后催化剂的 $\mathrm{F}$ 表面浓度可以看出, 反应后 F没有流失迹象.

图4为反应前催化剂Mo $3 d$ 的XPS谱图, 结合能 列于表4; 图5为反应后催化剂Mo $3 d$ 的XPS谱图及 分峰结果, 各分峰归属和相对量见表5. 从表4、5可 以看出, 反应前后催化剂 $\mathrm{Mo}(\mathrm{VI})$ 的结合能基本没有

表2 反应前催化剂的表面元素组成

Table 2 Surface element contents of the catalysts before reaction

\begin{tabular}{|c|c|c|c|c|c|c|c|c|}
\hline \multirow{2}{*}{ Catalyst } & \multicolumn{7}{|c|}{$x / \%$} & \multirow{2}{*}{$n(\mathrm{Ni}) / n(\mathrm{Ni}+\mathrm{Mo})$} \\
\hline & $\mathrm{C}$ & $\mathrm{O}$ & $\mathrm{Ni}$ & Mo & $\mathrm{Al}$ & $\mathrm{Ti}$ & $\mathrm{F}$ & \\
\hline CAT1 & 39.93 & 39.51 & 0.92 & 1.25 & 16.53 & 1.58 & 0.00 & 0.424 \\
\hline CAT2 & 34.71 & 42.28 & 1.30 & 1.34 & 16.46 & 1.78 & 2.12 & 0.492 \\
\hline CAT3 & 31.78 & 42.65 & 1.55 & 1.44 & 18.10 & 2.03 & 2.45 & 0.518 \\
\hline CAT4 & 19.37 & 51.55 & 1.41 & 1.75 & 21.64 & 1.74 & 2.72 & 0.446 \\
\hline
\end{tabular}

表3 反应后催化剂的表面元素组成

Table 3 Surface element contents of the catalysts after reaction

\begin{tabular}{ccccccccc}
\hline \multirow{2}{*}{ Catalyst } & \multicolumn{7}{c}{$x / \%$} & \\
\cline { 2 - 7 } & $\mathrm{C}$ & $\mathrm{O}$ & $\mathrm{Ni}$ & $\mathrm{Mo}$ & $\mathrm{Al}$ & $\mathrm{Ti}$ & $\mathrm{F}$ & \\
\hline CAT1 & 29.86 & 43.53 & 1.07 & 1.57 & 21.97 & 2.02 & 0.00 & 0.405 \\
CAT2 & 18.54 & 49.81 & 1.36 & 1.95 & 22.30 & 2.83 & 3.21 & 0.411 \\
CAT3 & 17.82 & 49.28 & 1.65 & 1.97 & 23.15 & 3.03 & 3.10 & 0.456 \\
CAT4 & 17.40 & 51.55 & 1.45 & 1.67 & 22.80 & 2.17 & 2.97 & 0.465 \\
\hline
\end{tabular}

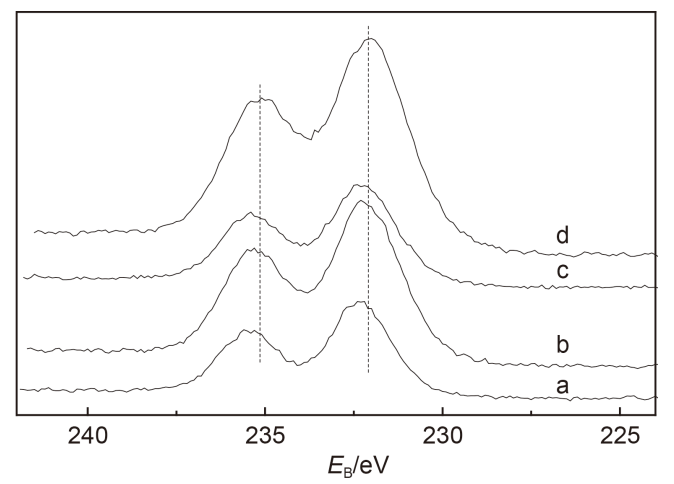

图4 反应前催化剂Mo 3d的XPS谱图

Fig.4 Mo $3 d$ XPS spectra of the catalysts before reaction (a) CAT1; (b) CAT2; (c) CAT3; (d) CAT4
变化, 其中CAT4的结合能较其它 3 个催化剂略小,说 明 $\mathrm{Mo}(\mathrm{VI})$ 物种与载体的相互作用进一步减弱, 有利 于Mo的硫化. 众所周知, $\mathrm{MoS}_{2}$ 是加氢脱硫催化剂的 活性组分, Mo物种的硫化程度是催化剂具有高活性 的前提. ${ }^{23,24}$ 从表 5 可以看出, 加F后催化剂中 $\mathrm{Mo}(\mathrm{IV})$ 占 $\mathrm{Mo}$ 物种的比例升高, 即 $\mathrm{F}$ 的加入使催化剂含有更 多的 $\mathrm{MoS}_{2}$ 相, 其中CAT4的 $\mathrm{MoS}_{2}$ 相浓度最高, 可达 $32.6 \%$. 这与 TPR表征结果一致.

\subsubsection{HRTEM表征}

图6(A, B) 分别为 4 个催化剂反应前后典型的 HRTEM电镜图. 从图6(A)可以看出, 氟改性催化剂 中 $\mathrm{Ni}$ 粒子颗粒变小, 分散性有所改善, 活性组分 $\mathrm{Mo}$ 与载体上的 $\mathrm{OH}$ 基团相互作用形成 $\mathrm{Mo}$ 氧化物单 层结构, 均匀的Mo氧化物单层结构无法在TEM照 片上形成祄度, 所以氧化态催化剂的电镜图未能提 供活性组分Mo氧化物的有效信息. 而反应后Mo组 分被硫化, 在电镜图中可以清晰地看见不同堆垛层 数且长短不同的 $\mathrm{MoS}_{2}$ 活性相(图中所示的黑线层状 结构即为 $\mathrm{MoS}_{2}$ 相. 从图6(B)中可以看出, 反应过程 中生成的 $\mathrm{MoS}_{2}$ 相在 4 个催化剂表面均分散的较为均 匀, 没有出现活性组分的聚集现象. 许多研究表 明,,$^{18,25} \mathrm{MoS}_{2}$ 相的堆垛层数及片晶长度是催化剂加氢 脱硫的关键. 为此, 本文选取了催化剂不同部位的 20张HRTEM照片, 大概200-300个 $\mathrm{MoS}_{2}$ 颗粒, 依据 公式(1)和(2)进行了统计分析, ${ }^{26}$ 结果列于图7和表6.

平均堆垛层数: $\quad \bar{N}=\frac{\sum N_{i} n_{i}}{\sum n_{i}}$

平均长度: $\quad \bar{L}=\frac{\sum L_{i} n_{i}}{\sum n_{i}}$

其中, $N$ 和 $L$ 分别为 $\mathrm{MoS}_{2}$ 片晶的平均堆垛层数及平 均长度; 公式(1)中 $N_{i}$ 为第 $i$ 个片晶的层数, $n_{i}$ 为层数为 $N_{i}$ 的片晶数; 公式(2)中 $L_{i}$ 为第 $i$ 个片晶的长度, $n_{i}$ 为长 度为 $L_{i}$ 的片晶数.

从图7(A)可以看出, 催化剂CAT1、CAT2、CAT3 中 $\mathrm{MoS}_{2}$ 相的堆垛层数主要集中在2-3层, 而CAT4集

表4 反应前催化剂Mo $3 d$ 的结合能

Table 4 Binding energies of before catalysts reaction

\begin{tabular}{lll}
\hline \multirow{2}{*}{ Catalyst } & \multicolumn{2}{c}{$E_{\mathrm{B}}(\mathrm{Mo} 3 d) / \mathrm{eV}$} \\
\cline { 2 - 3 } & $3 d_{5 / 2}$ & $3 d_{3 / 2}$ \\
\hline CAT1 & 232.3 & 235.4 \\
CAT2 & 232.3 & 235.4 \\
CAT3 & 232.3 & 235.3 \\
CAT4 & 232.1 & 235.2 \\
\hline
\end{tabular}



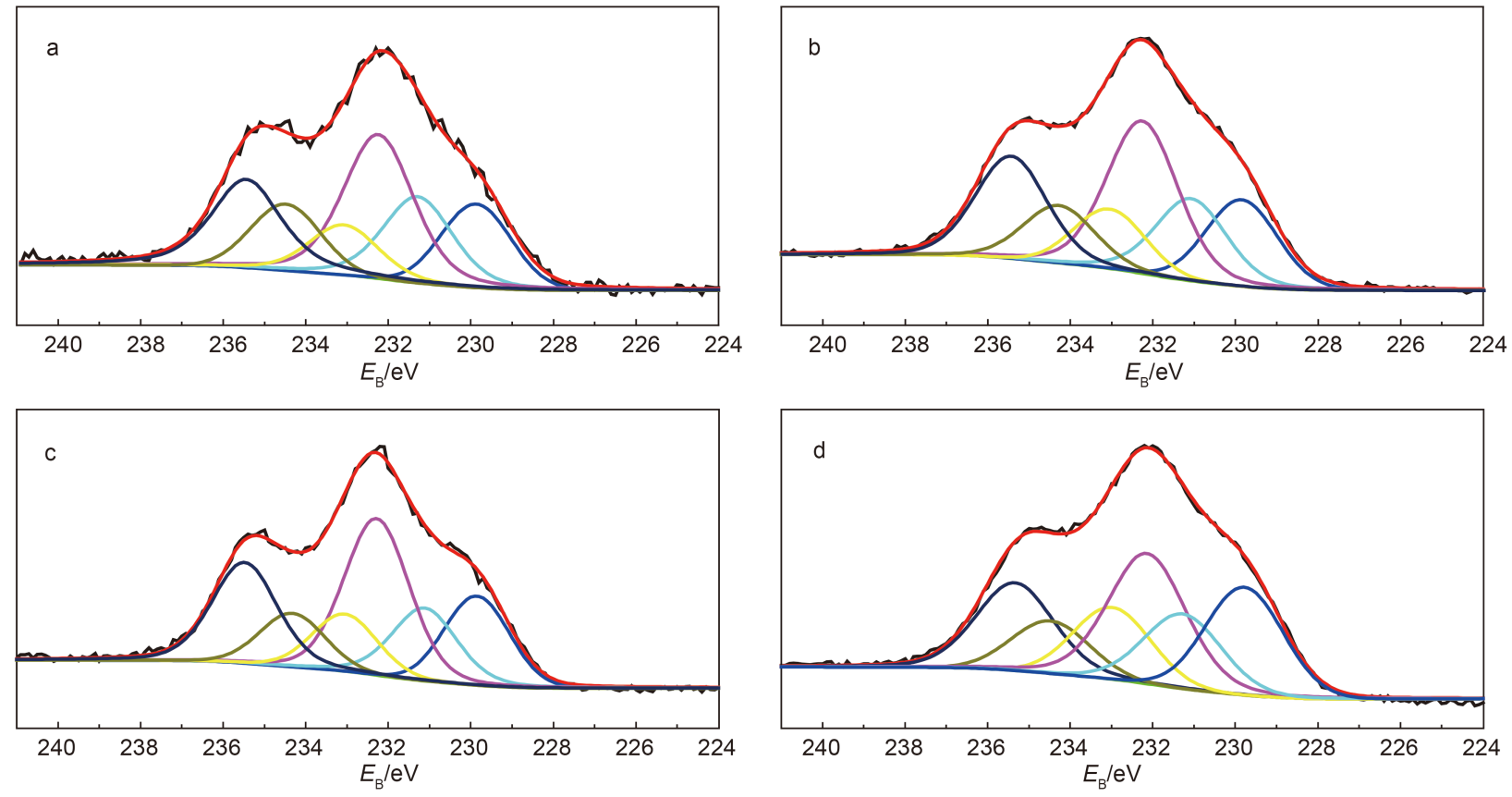

图5 反应后催化剂Mo 3d的XPS谱图

Fig.5 Mo $3 d$ XPS spectra of the catalysts after reaction

(a) CAT1; (b) CAT2; (c) CAT3; (d) CAT4

表5 催化剂中Mo 3d各物种的XPS参数

Table 5 XPS parameters of the different contributions of Mo $3 d$ of catalysts

\begin{tabular}{lllllc}
\hline \multirow{2}{*}{ Catalyst } & \multicolumn{3}{c}{$E_{\mathrm{B}} / \mathrm{eV}$} & & $x / \%$ \\
\cline { 2 - 3 } \cline { 5 - 5 } & $\mathrm{Mo}(\mathrm{IV})$ & $\mathrm{Mo}(\mathrm{V})$ & $\mathrm{Mo}(\mathrm{VI})$ & & $\mathrm{Mo}(\mathrm{IV}) /(\mathrm{Mo}(\mathrm{IV})+\mathrm{Mo}(\mathrm{V})+\mathrm{Mo}(\mathrm{VI}))$ \\
\hline CAT1 & 229.9 & 231.2 & 232.2 & 0.239 \\
CAT2 & 229.9 & 231.1 & 232.3 & 0.254 \\
CAT3 & 229.8 & 231.1 & 232.3 & 0.263 \\
CAT4 & 229.8 & 231.2 & 232.1 & 0.326 \\
\hline
\end{tabular}

中在3-4层. 与不加 $\mathrm{F}$ 的催化剂相比, 加 $\mathrm{F}$ 后催化剂单 层结构 $\mathrm{MoS}_{2}$ 的比例减少, 4 层结构 $\mathrm{MoS}_{2}$ 数量增多,

(A) (a)

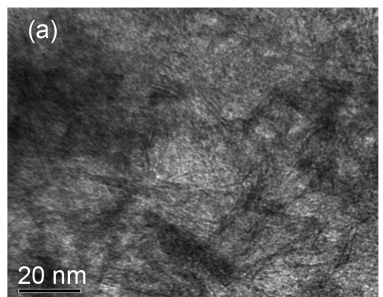

(B)

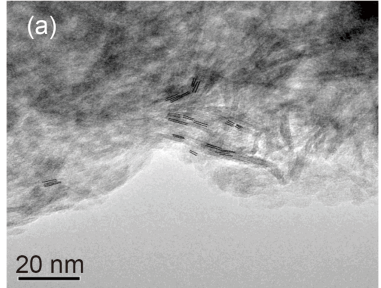

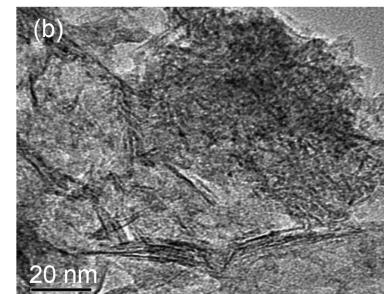

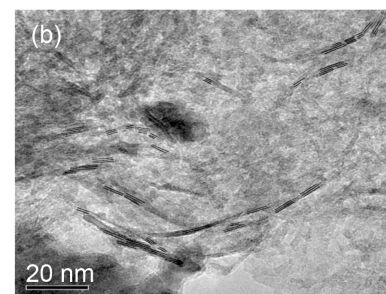

CAT3 和CAT4还出现一些5层结构的 $\mathrm{MoS}_{2}$. 由图 7(B) 可以看出, CAT1中 $\mathrm{MoS}_{2}$ 晶片长度主要集中在 4-6 nm, 而加F催化剂CAT2、CAT3、CAT4主要集 中在3-5 nm. 由表6看出, 加 $\mathrm{F}$ 后催化剂的平均堆垛 层数及片晶长度均有所增加, 其中 CAT4的平均堆 垛层数最大, 进一步证实了CAT4的加F方式更大程 度地削弱了 $\mathrm{Mo}$ 与载体的相互作用, 使 $\mathrm{MoS}_{2}$ 更容易 堆垛. $\mathrm{MoS}_{2}$ 在载体表面形成较大的活性堆垛, 对于 有取代基的二苯并噻吩类反应物克服空间位阻吸 附在活性中心位上非常有利. 结合前文XPS和TPR
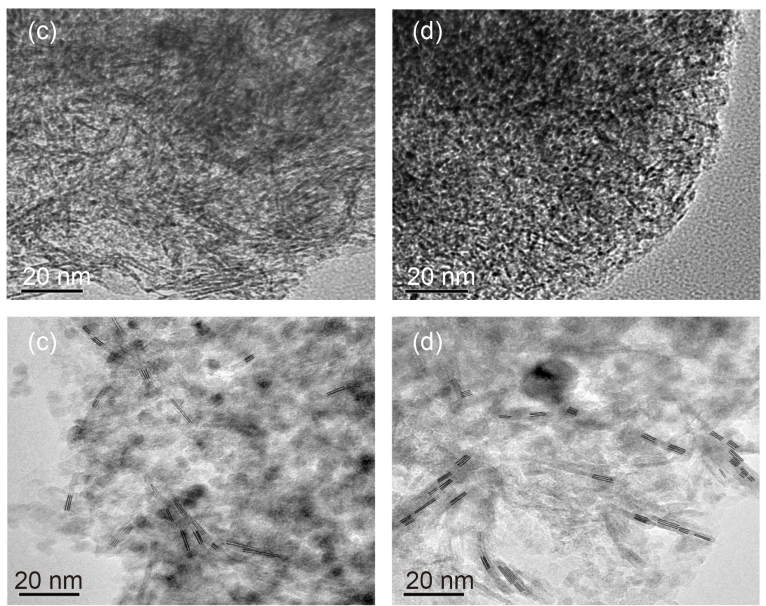

图6 催化剂反应前(A)后(B)高分辨透射电镜图

Fig.6 HRTEM images of catalysts before $(A)$ and after $(B)$ reaction

(a) CAT1; (b) CAT2; (c) CAT3; (d) CAT4 

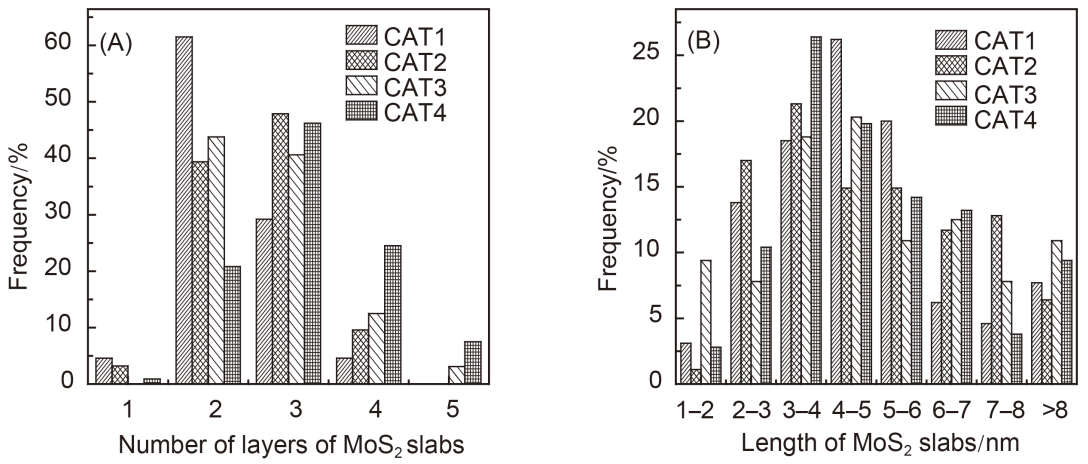

图7 催化剂反应后 $\mathrm{MoS}_{2}$ 晶粒的堆垛层数(A)及长度 $(\mathrm{B})$ 分布

Fig.7 Layer stacking (A) and length (B) distributions of $\mathrm{MoS}_{2}$ crystallites after reaction of catalysts

表6 催化剂反应后 $\mathrm{MoS}_{2}$ 晶粒的平均堆垛层数及平均长度

Table 6 Average length and number of layers of $\mathrm{MoS}_{2}$ phase in catalysts after reaction

\begin{tabular}{ccc}
\hline Catalyst & Average length/nm & Average stacking number \\
\hline CAT1 & 4.78 & 2.3 \\
CAT2 & 5.02 & 2.6 \\
CAT3 & 5.04 & 2.8 \\
CAT4 & 4.99 & 3.2 \\
\hline
\end{tabular}

表7 催化剂对4,6-DMDBT加氢脱硫的催化活性

Table 7 Catalytic activity in hydrodesulfurization (HDS) reaction of 4,6-DMDBT

\begin{tabular}{cccc}
\hline Catalyst & $w(\mathrm{~S})_{\mathrm{a}} /\left(\mu \mathrm{g} \cdot \mathrm{g}^{-1}\right)$ & $w(\mathrm{~S})_{\mathrm{b}} /\left(\mu \mathrm{g} \cdot \mathrm{g}^{-1}\right)$ & $x / \%$ \\
\hline CAT1 & 374.4 & 22.7 & 93.94 \\
CAT2 & 374.4 & 28.9 & 92.28 \\
CAT3 & 374.4 & 15.6 & 95.83 \\
CAT4 & 374.4 & 11.8 & 96.85 \\
\hline
\end{tabular}

表征可以结论, CAT4中含有更多的高活性II类NiMo-S活性相和适宜的堆垛层数 (3-5层最好) 是其活 性高的重要原因. ${ }^{27,28}$

\section{2 催化剂评价}

表7为催化剂对4,6-DMDBT加氢脱硫的催化活 性速度数据. 从表 7 可以看出, 4 个催化剂均对 $4,6-$ DMDBT有很高的脱硫活性，脱硫率都在 $90 \%$ 以上， 表明通过在催化剂中引入F可以使催化剂性能稳定 再现，进而使完全液相技术更好地应用于柴油超精 脱硫催化剂的制备. 从催化剂的脱硫活性排序 CAT4 $>$ CAT3 $>$ CAT $1>$ CAT2来看, 有益的方 式是在 $\mathrm{Ni} 、 \mathrm{Mo}$ 之前引入 $\mathrm{F}$, 最佳的方式是在此前提 下不使用浓 $\mathrm{HNO}_{3}$ 作水解催化剂, 此时脱硫率最高, 可达 $96.85 \%$. 可以推断, 由于HF酸是弱酸, 在强酸浓 $\mathrm{HNO}_{3}$ 的存在下, 引入的 $\mathrm{F}^{-}$离子因生成 $\mathrm{HF}$ 而失去电 性, 进而影响其落位, 导致其有益的作用减弱.
4 结 论

通常认为, 4,6-DMDBT由于有较大的空间位阻, 其脱硫过程主要是通过HYD, 所以提高催化剂的加 氢活性成为关键; ${ }^{29,30}$ 催化剂表面的金属 $\mathrm{Ni}$ 及 II 类 $\mathrm{Ni}$ Mo-S活性相可以提高催化剂的加氢活性和 $\mathrm{C}-\mathrm{S}$ 键 的氢解能力, 从而对 4,6-DMDBT类大分子化合物有 较高的加氢脱硫活性. ${ }^{17,31-33}$ 本文研究表明, 采用完全 液相法制备柴油超精加氢脱硫催化剂, 在活性组分 加入前及无浓 $\mathrm{HNO}_{3}$ 存在下引入 $\mathrm{F}$, 可以最有效地分 散加氢活性组分 $\mathrm{Ni}^{0}$, 并防止在反应过程中聚集, 从 而保证催化剂性能的稳定再现. 另外, F的引入还能 够削弱 $\mathrm{Ni} 、 \mathrm{Mo}$ 与载体的相互作用, 生成易于硫化的 聚集态Mo-O-Mo, 并使Ni在其中很好地分散, 进而 促进了更多的适宜堆垛层数和长度的II类Ni-Mo$\mathrm{S}$ 活性相的生成, 以及具有更好的整体织构参数, 从 而使脱硫活性显著增加.

\section{References}

(1) Stanislaus, A.; Marafi, A.; Rana, M. S. Catal. Today 2010, 153, 1. doi: $10.1016 /$ j.cattod.2010.05.011

(2) Chen, Y. D.; Wang, L.; Liu, T. F.; Zhang, Y. L.; Liu, X. Y.; Jiang, Z. X.; Li, C. Chem. React. Eng. Technol. 2013, 29, 392. [陈燕蝶, 王 璐, 刘铁峰. 张玉良, 刘欣毅, 蒋宗轩, 李 灿. 化 学反应工程与工艺, 2013, 29, 392.]

(3) Si, Y. H.; Zhu, Y. Q.; Zou, R. M.; Guo, F.; Wang, J. C. Chem. Eng. Oil Gas. 2014, 43, 82. [司云航, 朱玉琴, 邹蓉梅. 郭 凡, 王嘉春. 石油与天然气化工, 2014, 43, 82.]

(4) Mochida, I.; Choi, K. J. Jpn. Pet. Inst. 2004, 47, 145. doi: 10.1627/jpi.47.145

(5) Song, C. Catal. Today 2003, 86, 211. doi: 10.1016/S09205861(03)00412-7

(6) Matos, E. M.; Guirardello, R.; Mori, M.; Nunhez, J. R. Comput. Chem. Eng. 2009, 33, 1115. doi: 10.1016/j. compchemeng.2008. 12.011 
(7) Deng, Z. H.; Wang, T. F.; Wang, Z. W. Chem. Eng. Sci. 2009, $65,480$.

(8) Gao, Z. H.; Hao, L. F.; Huang, W.; Xie, K. C. Catal. Lett. 2005, 102, 139. doi: 10.1007/s10562-005-5845-7

(9) Gao, Z. H.; Huang, W.; Hao, L. F.; Xie, K. C. Chin. J. Catal. 2006, 27, 86. [高志华, 黄 伟, 郝利峰, 谢克昌. 催化学报, 2006, 27, 86.]

(10) Li, Z. B. Complete Liquid-Phase Technology for Ultra Deep Hydrodesulfurizationin in Simulation Diesel. MS. Dissertation, Taiyuan University of Technology, Taiyuan, 2014. [李志博. 完 全液相催化剂制备技术在模拟柴油超精脱硫中的应用研究 [D]. 太原: 太原理工大学, 2014.]

(11) Lü, J.; Zhang, W.; Yang, Z. Q.; Li, J. Y.; Tang, X. B.; Lü, J. Chem. World 2012, 9, 567. [吕 婧, 张 伟, 杨志强, 李娇毅, 唐晓博, 吕 剑. 化学世界, 2012, 9, 567.]

(12) Kim, H.; Lee, J. J.; Moon, S. H. Appl. Catal. B 2003, 44, 287. doi: 10.1016/S0926-3373(03)00077-8

(13) Sun, M. Y.; Nicosia, D.; Prins, R. Catal. Today 2003, 86, 173. doi: 10.1016/S0920-5861(03)00410-3

(14) Matralis, H.; Papadopoulou, C.; Lycourghiotis, A. Appl. Catal. A-Gen. 1994, 116, 221. doi: 10.1016/0926-860X(94)80291-2

(15) Lewis, J. M.; Kydd, R. A.; Boorman, P. M. J. Catal. 1989, 120, 413. doi: 10.1016/0021-9517(89)90281-9

(16) Sarbak, Z.; Andersson, S. L. T. Appl. Catal. 1991, 69, 235. doi: 10.1016/S0166-9834(00)83305-9

(17) Eliche-Quesada, D.; Merida-Robles, J.; Maireles-Torres, P.; Rodriguez-Castellon, E.; Jimenez-Lopez, A. Langmuir 2003, 19, 4985. doi: 10.1021/la0208651

(18) Lai, W. K.; Pang, L. Q.; Zheng, J. B.; Li, J. J.; Wu, Z. F.; Yi, X. D.; Fang, W. P.; Jia, L. S. Fuel. Process. Technol. 2013, 110, 8. doi: 10.1016/j.fuproc.2013.01.006

(19) Liu, H.; Yin, C. L.; Li, H.; Liu, B.; Li, X. H.; Chai, Y. M.; Li, Y. P.; Liu, C. G. Fuel 2014, 129, 138. doi: 10.1016/j.fuel. 2014.03.055
(20) Qu, L. L.; Zhang, W. P.; Prins, R.; Kooyman, P. J. J. Catal. 2003, 215, 7. doi: 10.1016/S0021-9517(02)00181-1

(21) Cuevas, R.; Ramirez, J.; Busca, G. J. Fluorine Chem. 2003, 122 , 151. doi: 10.1016/S0022-1139(03)00054-X

(22) Al-Dalama, K.; Stanislaus, A. Thermochim. Acta 2011, 520, 67. doi: 10.1016/j.tca.2011.03.017

(23) Damyanova, S.; Petrov, L.; Grange, P. Appl. Catal. A-Gen. 2003, 239, 241. doi: 10.1016/S0926-860X(02)00385-X

(24) Rashidi, F.; Sasaki, T.; Rashidi, A. M.; Nemati K. A.; Jozani, K. J. J. Catal. 2013, 299, 321.. doi: 10.1016/j.jcat.2012.11.012

(25) Li, H. F.; Li, M. F.; Chu, Y.; Liu, F.; Nie, H. Appl. Catal. A-Gen. 2011, 403, 75. doi: 10.1016/j.apcata.2011.06.015

(26) Yao, S. D.; Zheng, Y.; Ng, S.; Ding, L. H.; Yang, H. Appl. Catal. A-Gen. 2012, 435-436, 61.

(27) Shimada, H. Catal. Today 2003, 86, 17. doi: 10.1016/S09205861(03)00401-2

(28) Guo, R.; Shen, B. X.; Fang, X. C.; Sun, J.; Peng, C.; Cui, X. L. China Pet. Process. Pe. 2014, 16, 12.

(29) Sanchez-Minero F.; Ramirez, J.; Gutierrez-Alejandre, A.; Fernandez-Vargasa, C.; Torres-Manceraa, P.; Cuevas-Garciaa, R. Catal. Today 2008, 133-135, 267.

(30) Manoli, J. M.; Costa, P. D.; Brun, M.; Vrinat, M.; Mauge, F.; Potvin, C. J. Catal. 2004, 221, 365. doi: 10.1016/j.jcat. 2003.08.011

(31) Zhang, Z. C.; Huang, X. L.; Wei, H. J.; Liu, J. J.; Liu, Z. Petrochem. Technol. 2009, 38, 1353. [张志成, 黄星亮, 魏焕景, 刘建军, 刘 志. 石油化工, 2009, 38, 1353.]

(32) Vradman, L.; Landau, M. V.; Herskowitz, M. Fuel 2003, 82, 633. doi: 10.1016/S0016-2361(02)00354-X

(33) Qihe, R. M.; Yuan, H.; Li, H. F.; Zhang, W. H.; Xu, G. T. Acta Petrolei. Sin.(Pet. Process. Sect.) 2011, 27, 429. [齐和日玛, 袁 蕙, 李会峰, 张暳宏, 徐广通. 石油学报 (石油加工), 2011, 27, 429.] 